\title{
SISTEMA DE INFORMACIÓN DE RIESGOS: FACTOR DE VISIBILIDAD
}

\author{
Risk information system: The visibility factor
}

\section{Fernando Vegas-Fernández}

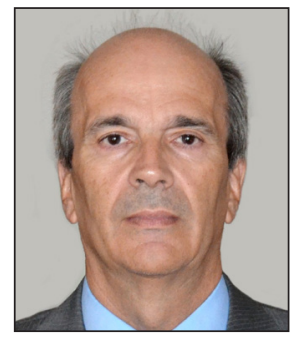

Fernando Vegas-Fernández es ingeniero de caminos por la Escuela Técnica Superior de Ingenieros de Caminos, Canales y Puertos de la Universidad Politécnica de Madrid. Ha sido director de sistemas de información y director de riesgos en empresas españolas del IBEX-35. Es miembro de Cionet y de asociaciones de riesgos (Agers y American Risk \& Insurance Association) y de seguridad (ASIS International). Prepara su tesis doctoral en la citada universidad, aunando su experiencia en sistemas y en riesgos.

http://orcid.org/0000-0003-1968-9891

Universidad Politécnica de Madrid Escuela Técnica Superior de Ingenieros de Caminos, Canales y Puertos Profesor Aranguren, 3. 28040 Madrid, España fvegas@ciccp.es

\section{Resumen}

La complejidad de la valoración de riesgos requiere sistemas de información que mejoren la objetividad y sean más visuales, intuitivos y flexibles. El estudio de varios miles de proyectos de construcción ha posibilitado el diseño de una solución que satisface estas exigencias gracias a un innovador indicador de riesgo: el Factor de visibilidad. Este indicador asigna valores cuantitativos para los riesgos y hace posible obtener un resumen de cualquier escenario de riesgo. La orientación del sistema propuesto es visual y analítica para facilitar la toma de decisiones, y posibilita conocer en todo momento el grado de riesgo para cualquier nivel de agregación. Se proponen plantillas para la introducción de los datos, con cuestionarios basados en una organización funcional de los riesgos, conteniendo preguntas y respuestas predefinidas cuyos pesos varían en función del contexto. El sistema, probado en una empresa constructora del IBEX-35, es aplicable a cualquier sector adaptando los cuestionarios.

\section{Palabras clave}

Riesgo; Indicadores de riesgo; Combinación de riesgos; Resumen de riesgos; Riesgo de proyecto; Visualización; Visualización de riesgos; Visualización de datos; Visualización de información; Diseño de información; Sistemas de información.

\section{Abstract}

Project risk evaluation complexity demands modern information systems that increase objectivity and are more visual, intuitive, and flexible. Thousands of construction projects have been analyzed to define a risk system that fulfills these requirements and uses a new kind of risk indicator: The Visibility factor. This indicator makes it possible to get quantitative values for any risk and to summarize risk scenarios. The system is analytical and the result is visual in order to allow the risk level to be identified at any stage, making it much easier for decision-makers to determine the best possible option. Risk data is collected via multiple choice questionnaires, built over a template, and the sections are weighted based on the project characteristics and its circumstances. This system can be used in any industry by modifying the questionnaires and it has been tested in a Spanish IBEX-35 construction company.

\section{Keywords}

Risk; Risk indicators; Risk combination; Risk summary; Project risk; Visualisation; Risk visualisation; Data visualisation; Information visualization; Information design; Information systems.

Vegas-Fernández, Fernando (2017). "Sistema de información de riesgos: Factor de visibilidad". El profesional de la información, v. 26, n. 6, pp. 1065-1075.

https://doi.org/10.3145/epi.2017.nov.06 


\section{Introducción}

Riesgo es la posibilidad de que se produzca un efecto adverso (Whittaker, 2015). El análisis de riesgos de proyectos, particularmente en los proyectos de construcción, se ha complicado mucho desde 2008 debido a la crisis económica -que ha obligado a las empresas a trabajar lejos de sus países de origen- y a las exigencias del marco regulatorio (Di-Marco; Taylor; Alin, 2010; Ferma/Eciia, 2014; Arjona, 2015; Seopan, 2015; MEC, 2016; Leung; Liang; Chan, 2017). Por otra parte, los riesgos son diversos (Mishra; Mishra, 2016), su identificación, análisis y descripción es difícil, y la información disponible para su valoración es parcial y subjetiva (Al-Bahar; Crandall, 1990).

Un riesgo se define básicamente por su impacto y la probabilidad de que acontezca (Steinberg et al., 2004; Whittaker, 2015), pero es frecuente utilizar indicadores adicionales para mejorar su identificación (Aenor, 2011; Curtis; Carey, 2012) y adaptarse a los riesgos emergentes (World Economic Forum, 2015; 2016; 2017). Sin embargo, un riesgo necesita ser medido para ser comprendido (The Joint Forum, 2003; Babatola, 2004; The Institute of Risk Management, 2011; 2012). Las empresas diseñan sistemas de información de riesgos cada vez más complicados, aunque frecuentemente su cultura de riesgos es baja (Zou; Chen; Chan, 2010), por lo que son difíciles de manejar y no siempre proporcionan la información que necesitan los directivos para sus decisiones.

La evaluación de riesgos demanda sistemas de información que mejoren la objetividad y sean más visuales, intuitivos y flexibles

Las consecuencias de la materialización de un riesgo son en primera instancia un sobrecoste o un retraso, pero el impacto también puede ser de reputación, seguridad, calidad, salud, medio ambiente o cualquier combinación de los anteriores. El número de riesgos a considerar puede ser muy alto. Para un proyecto de construcción cabría distinguir al menos 12 orígenes del riesgo, como país, financiación, logística, etc. (Mishra; Mishra, 2016), pudiendo contemplarse en cada uno hasta 10 aspectos. Por ejemplo, para el origen del riesgo país, cabe considerar los aspectos político, económico, de seguridad, climatológico, salud, etc. Para cada aspecto se podrían plantear hasta 10 preguntas relativas al riesgo, cada una con diversas respuestas posibles. Esto significaría potencialmente 33.600 riesgos (considerando 4 posibles respuestas y los 7 tipos de impacto indicados), aunque en la práctica son bastantes menos.

La comunicación del grado de riesgo a la dirección de la empresa es esencial para que ésta lo contraste con su apetito de riesgo (Al-Bahar; Crandall, 1990; Steinberg et al., 2004; Airmic/Alarm/IRM, 2010; Beasley; Branson; Hancock, 2010). La información comunicada debe ser clara, objetiva y fácilmente comprensible, lo cual no siempre es fácil debido al gran volumen de datos, la subjetividad y las presiones internas. Resumir la situación de riesgo total supone una dificultad adicional.
En esta investigación se han examinado los informes de riesgo de los proyectos de construcción de una empresa española del IBEX-35 con presencia internacional: constaban de más de 40 páginas de texto por cada proyecto, y los informes ejecutivos de riesgo con los proyectos valorados tenían 20 páginas, sin ningún indicador resumen de la situación de riesgo total, por país o por proyecto. Cabría aplicar aquí el enfoque de "extracción de estructuras de grandes conjuntos de datos que puedan ser representadas de forma visual a los usuarios" y el planteamiento de visualización y de captura de las cualidades esenciales de los datos (Olmeda-Gómez, 2014).

Se han tenido en cuenta anteriores trabajos para buscar un indicador único, sencillo, lineal e intuitivo que facilite la comunicación de una situación de riesgo (Pont-Sorribes; Codina; Pedraza-Jiménez, 2009; Cretu; Stewart; Berends, 2011). Ejemplos como el de la información de oleaje (escala de Douglas), de viento (escala de Beaufort), de peligro de aludes (escala europea de 1993) o de seísmos (escala de Richter) ilustran el deseo de simplificar la información de riesgo.

Se han considerado los aspectos relativos a la objetividad de la información, apetito al riesgo y subjetividad en la percepción del riesgo, así como la influencia de políticas internas o estrategias de empresa que distorsionen la realidad del riesgo identificado (Pratt, 1964; De-Frutos-Torres; Marcos-Santos, 2017; Vilajoana-Alejandre; Rom-Rodríguez, 2017).

\section{Objeto y método}

El sistema de información de riesgos de proyectos debe ser claro, escueto y ágil (Cretu; Stewart; Berends, 2011). La fiel comunicación del conocimiento obtenido por los evaluadores de riesgos de un proyecto, su compartición y el acceso rápido al mismo se han considerado requisitos esenciales, así como el aumento de la objetividad mediante la automatización. La búsqueda de indicadores visuales e intuitivos y la simplificación, sustituyendo texto por datos o gráficos para facilitar la comprensión, han sido permanentes en esta investigación (Lundy, 2012; Olmeda-Gómez, 2014).

Como referencia se examinó en profundidad la forma de estudiar los riesgos de proyectos de la empresa referida anteriormente, y se cotejó la información obtenida con otras empresas nacionales y extranjeras, directamente mediante reuniones o a través de consultores expertos en análisis de riesgos.

El análisis de sus métodos de trabajo y sus carencias reveló estos inconvenientes:

- Uso de narración para describir los riesgos: dificultad de correcta interpretación y traducción;

- Incapacidad de resumir riesgos, habiendo cientos de ellos de los que no se conoce su resumen;

- Carencia de un indicador de riesgo cuantitativo lineal e intuitivo: la diversidad de riesgos dificulta la aplicación de indicadores específicos a tipos de riesgo diferentes, el coste no siempre es calculable, y la esperanza matemática (impacto x probabilidad) proporciona un resultado cuya valoración requiere el uso de tablas de interpretación (Project Management Institute, 2013). 
Con los requisitos y obstáculos antes descritos se concretó el objeto de la investigación:

- Definición de un indicador de riesgo cuantitativo genérico, automático, intuitivo, lineal y visual: formulación matemática a partir de la definición cualitativa del impacto y la probabilidad;

- Definición de un sistema de combinación de eventos de riesgo: el resultado es un evento resumen con impacto y probabilidad propios;

- Conversión de las valoraciones cualitativas de riesgo en valores cuantitativos;

- Definición de cuestionarios: preguntas, respuestas y pesos;

- Definición de campos a cumplimentar en los formularios;

- Diseño de formatos: formularios de entrada y gráficos, pantallas interactivas e informes de salida;

- Planteamiento alternativo para la valoración del coste del riesgo;

- Sistema de información de riesgos de proyecto: informatizado y basado en cuestionarios.

El método investigador incluyó trabajos de gabinete, reuniones de expertos, entrevistas, tormenta de ideas, y método Delphi.

La orientación del sistema propuesto es visual y analítica para facilitar la toma de decisiones

En gabinete se estudiaron las recomendaciones de actuación de organismos como el Project Management Institute (Schuyler, 2001; Project Management Institute, 2013), Committee of Sponsoring Organizations of the Treadway Commission (COSO, 2004; Steinberg et al., 2004; Beasley; Branson; Hancock, 2010; Curtis; Carey, 2012) o Aenor (Aenor, 2010; 2011).

Al no hallarse un indicador con las propiedades buscadas y con capacidad de combinación recurrente de grupos de eventos de riesgo, se acometió su definición matemática. Posteriormente se aplicaron los resultados a proyectos reales y se contrastaron los resultados con expertos funcionales.

Para construir los cuestionarios se siguió un método mixto con entrevistas, método Delphi, juicio de expertos y posterior validación en común. Se pidió a los departamentos funcionales de la empresa que elaborasen sus propios cuestionarios, que fueron posteriormente revisados por otros departamentos y expertos externos. Se pusieron en funcionamiento y se repitió el proceso varias veces en un plazo de dos años, hasta lograr la estabilidad de los cuestionarios.
El contexto de esta investigación fue el de proyectos de construcción, pero se procuró que los resultados -salvo los cuestionarios- fuesen aplicables en cualquier entorno.

\section{Resultados}

Dado que los fines eran variados, se trabajó en líneas independientes que debían converger en una aplicación informática. El producto final se implantó con éxito en la empresa constructora antes referida y recibió la aprobación de evaluadores, responsables y consultores externos. Los resultados se presentan de acuerdo con estas líneas de actuación:

- programación de la aplicación informática;

- definición del indicador de riesgo y de los algoritmos de combinación;

- definición de los cuestionarios;

- definición de campos;

- definición de la lógica de introducción de datos;

- definición del sistema de valoración del coste de los riesgos.

\subsection{Aplicación informática}

Se revisaron numerosas aplicaciones comerciales, que no cumplían los requisitos especificados, y se optó por reprogramar una de ellas para lograr cumplirlos. La programación en sí carece de interés para este artículo y sólo se destacarán los aspectos relevantes.

Los sistemas de información de riesgos no siempre proporcionan la información que necesitan los directivos para sus decisiones

\subsubsection{Diseño}

La cantidad de información a manejar era tal que el diseño de pantallas supuso un reto para lograr eficacia en su uso. Se consideraron dos pantallas principales: la de proyectos y la de valoración de riesgos de proyecto (cuestionarios).

La primera recoge la lista de proyectos con sus datos básicos y su información de riesgo en cada uno de los 10 cuestionarios utilizados, con valores e indicadores de color (figura 1).

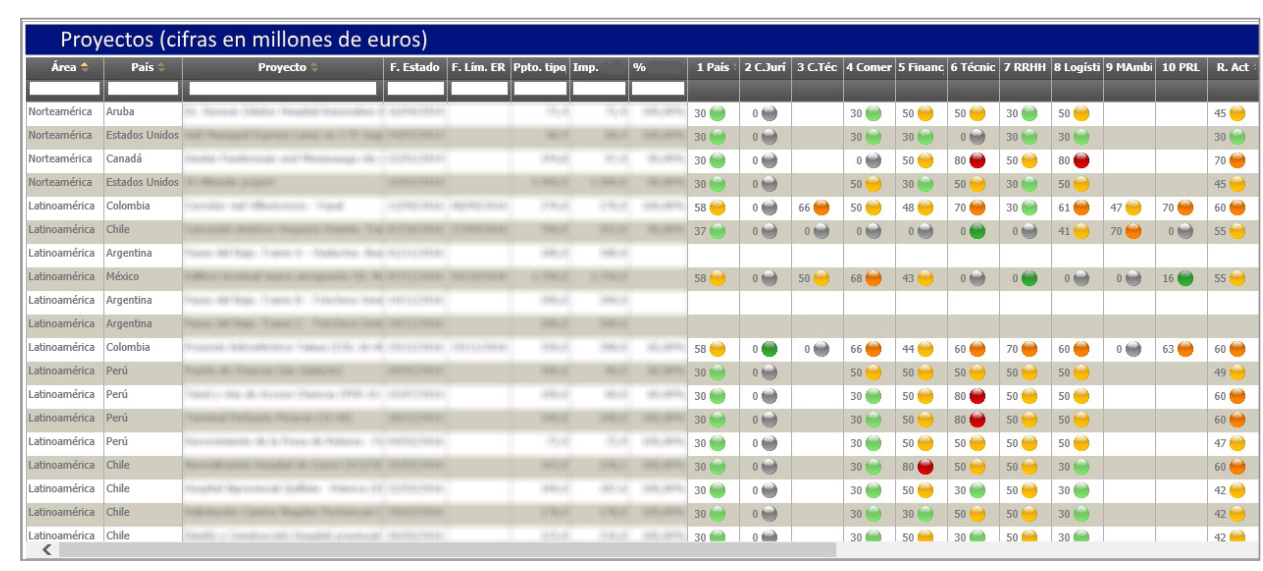

Figura 1. Pantalla de proyectos (datos ficticios) 
La segunda pantalla es la utilizada por los evaluadores de riesgos para definir y revisar los riesgos (figura 2).

Esta pantalla presenta las preguntas del cuestionario organizadas jerárquicamente. El ejemplo corresponde a la sección 5 del cuestionario de riesgos técnicos. Las preguntas se muestran junto con sus posibles respuestas. Al seleccionar una respuesta diferente de "No hay riesgo" se activan los campos presentes y emergen otros adicionales para añadir información. El evaluador usa el

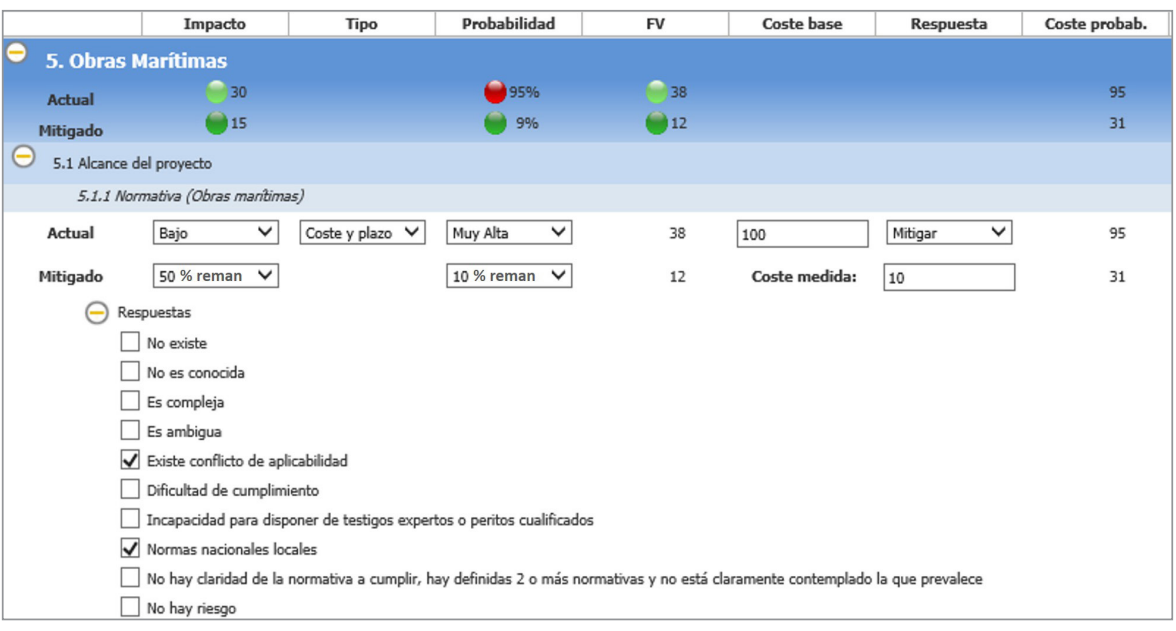

Figura 2. Plantilla de valoración de riesgos mediante un cuestionario ratón y no escribe -salvo en dos

campos descriptivos-, por lo que la información guardada no es texto sino datos y se puede mostrar en cualquier idioma. En función de la identificación y valoración de los riesgos se actualiza automáticamente la valoración global de riesgo de la sección, visible en la fila superior.

\subsubsection{Conexión con la base de datos de proyectos}

La base de datos de proyectos y la de riesgos deben ser compartidas para que al darse de alta un proyecto, el sistema de riesgos lo detecte automáticamente y pueda decidirse si deben considerarse o no los riesgos. Así se gana en agilidad y se evitan errores.

\section{La comunicación del grado de riesgo a la} dirección de la empresa es esencial para que ésta la contraste con su apetito de riesgo

\subsection{Indicador genérico de riesgo y algoritmos de combinación}

El indicador definido es el Factor de visibilidad del riesgo o Fv (abreviado, Factor de visibilidad).

La parte superior de la figura 2 muestra los datos resumen de la valoración global de riesgo de la sección estudiada: impacto, probabilidad, Fv y coste en las situaciones actual y mitigada. Estos ocho valores resumen la información de riesgo de esta sección. Se verá posteriormente que cada cuestionario consta de varias secciones y que éstas pueden contener subsecciones. Este esquema de ocho valores resumen se repite en los cuestionarios -agrupando todas sus secciones-, en los proyectos -resumiendo todos sus cuestionarios-, en los países -resumiendo todos sus proyectosy en el conjunto de la empresa. En cada caso el resumen se presenta como un simple evento de riesgo, con su impacto, probabilidad, Fv y coste.

El Factor de visibilidad Fv es el resultado de la investigación realizada para obtener un indicador lineal, intuitivo y fácil de comprender. Está concebido para conseguir una valoración cuantitativa automática de cualquier riesgo a partir de la va- loración cualitativa de su impacto y probabilidad -de modo que no suponga un trabajo extra para el evaluador y que el responsable pueda percibir eficazmente dónde hay riesgo y dónde no- y para lograr combinar riesgos, obteniendo un único evento de riesgo representativo.

El condicionante básico de este indicador es la visibilidad. Está orientado a la detección del riesgo priorizando la identificación sobre la exactitud, que no es posible alcanzar dado que se está tratando con estimaciones de riesgo. Este enfoque muestra una gran coincidencia con los atributos planteados por Olmeda-Gómez (2014) y Card, Mackinlay y Shneiderman (1999) en referencia a la visualización.

\subsubsection{Visualización}

El Factor de visibilidad origina curvas de nivel de riesgo en un gráfico bidimensional de impacto y probabilidad o mapa de calor (figura 3).

Puede apreciarse que las curvas están distribuidas uniformemente en el mapa, son simétricas respecto a la bisectriz del 2 o cuadrante (la diagonal que va del extremo inferior izquierdo al superior derecho) y toman su valor del punto del mapa en el que el impacto es igual a la probabilidad (multiplicada por 100). De ahí su fácil comprensión y manejo.

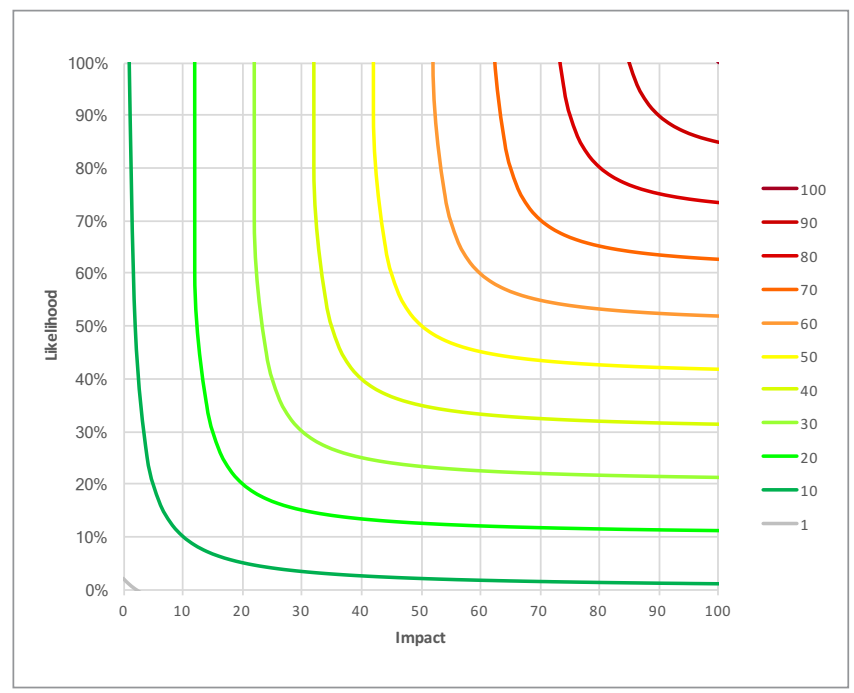

Figura 3. Curvas de igual riesgo del Factor de visibilidad 
La severidad de un riesgo, dados su impacto y probabilidad, se percibe inmediatamente viendo el mapa. Igualmente, conocido un valor de $F v$, se obtiene una rápida idea de su gravedad sin necesidad de mapa.

\subsubsection{Formulación de Factor de visibilidad Fv}

Su formulación matemática es el resultado de resolver unas simples ecuaciones para obtener las curvas anteriores. El resultado es:

$$
F v=\frac{100 p+20+i-\sqrt{10000 p^{2}+i^{2}-200 p i+400}}{2}
$$

\subsubsection{Escalas de valoración}

La aplicación de la fórmula anterior a las valoraciones cualitativas exige un proceso previo para convertir en números las valoraciones conceptuales de los evaluadores.

El evaluador debe indicar para cada riesgo cuál es su impacto y la probabilidad de que acontezca. Esto no siempre es posible numéricamente -particularmente en proyectos de construcción-, y se hace cualitativamente eligiendo un nivel dentro de un rango dado (Likert, 1932). Para ello el sistema presenta desplegables con 5 niveles de selección, desde muy bajo a muy alto (ver figura 2). A partir de esta elección la conversión es muy sencilla estableciendo una escala de asignación.

Tabla 1. Escala de asignación numérica a la valoración cualitativa

\begin{tabular}{|l|c|c|}
\hline \multicolumn{1}{|c|}{ Selección } & Rango & Valor \\
\hline Muy bajo & $0-20$ & 10 \\
\hline Bajo & $20-40$ & 30 \\
\hline Medio & $40-60$ & 50 \\
\hline Alto & $60-80$ & 70 \\
\hline Muy alto & $80-100$ & 95 \\
\hline
\end{tabular}

La escala elegida es lineal y simple. La presentación coloreada de las opciones (de verde a rojo) refuerza en el evaluador la visibilidad del mensaje a transmitir. Se han descartado otras posibles escalas de tipo no lineal o logarítmico porque confunden a los evaluadores, y se ha optado por la simplicidad.

Complementariamente a estas escalas de asignación se manejan escalas de interpretación. Éstas posibilitan calificar la gravedad de los valores de Fv obtenidos, distinguiéndolos con colores (ver figura 2) -también se puede aplicar a los impactos y probabilidades, aunque tiene menos sentido dado que son sólo componentes individuales del riesgo-. La escala de interpretación responde al apetito de riesgo de la empresa. Puede coincidir o no con la de asignación.

\subsubsection{Formulación de la combinación}

La obtención de un evento de riesgo que constituya el resumen de un escenario es un aspecto esencial de información de los riesgos de un proyecto. El pilar del método es el Factor de visibilidad.

En esencia se obtienen dos puntos de ajuste utilizando los valores medios ponderados de los impactos, probabilidades y Factores de visibilidad de todos los puntos. Estos dos puntos se promedian posteriormente para obtener el resultado final.

Siendo $I_{j}, P_{j}, F v_{j}, D_{j}$ los valores de impacto, probabilidad, Factor de visibilidad y peso de cada elemento individual, se calcula para cada uno un peso corregido $D_{j}^{\prime}$ :

$D_{j}^{\prime}=\left(F v_{j} \times D_{j}\right)^{2}$

Con este peso se calculan las medias:

$$
I_{m}=\frac{\sum_{j=1}^{n} I_{j} \times D^{\prime}{ }_{j}}{\sum_{j=1}^{n} D^{\prime}{ }_{j}} ; \quad P_{m}=\frac{\sum_{j=1}^{n} P_{j} \times D^{\prime}{ }_{j}}{\sum_{j=1}^{n} D^{\prime}{ }_{j}} ; \quad F v_{m}=\frac{\sum_{j=1}^{n} F v_{j} \times D^{\prime}{ }_{j}}{\sum_{j=1}^{n} D^{\prime}{ }_{j}}
$$

El punto de ajuste $I$ es el que tiene como impacto el valor $I_{m}$ y está en la curva $F v_{m}$. Su probabilidad es $P_{a}$.

El punto de ajuste $P$ es el que tiene como probabilidad el valor $P_{m}$ y está en la curva $F v_{m}$. Su impacto es $I_{a}$.

Dados los dos puntos anteriores se calculan sus valores medios:

$$
I_{m a}=\frac{I_{m}+I_{a}}{2}, \quad P_{m a}=\frac{P_{m}+P_{a}}{2}, \quad F v_{a}=F v\left(I_{m a}, P_{m a}\right)
$$

Finalmente se aplica la pequeña corrección necesaria para cuadrar los valores:

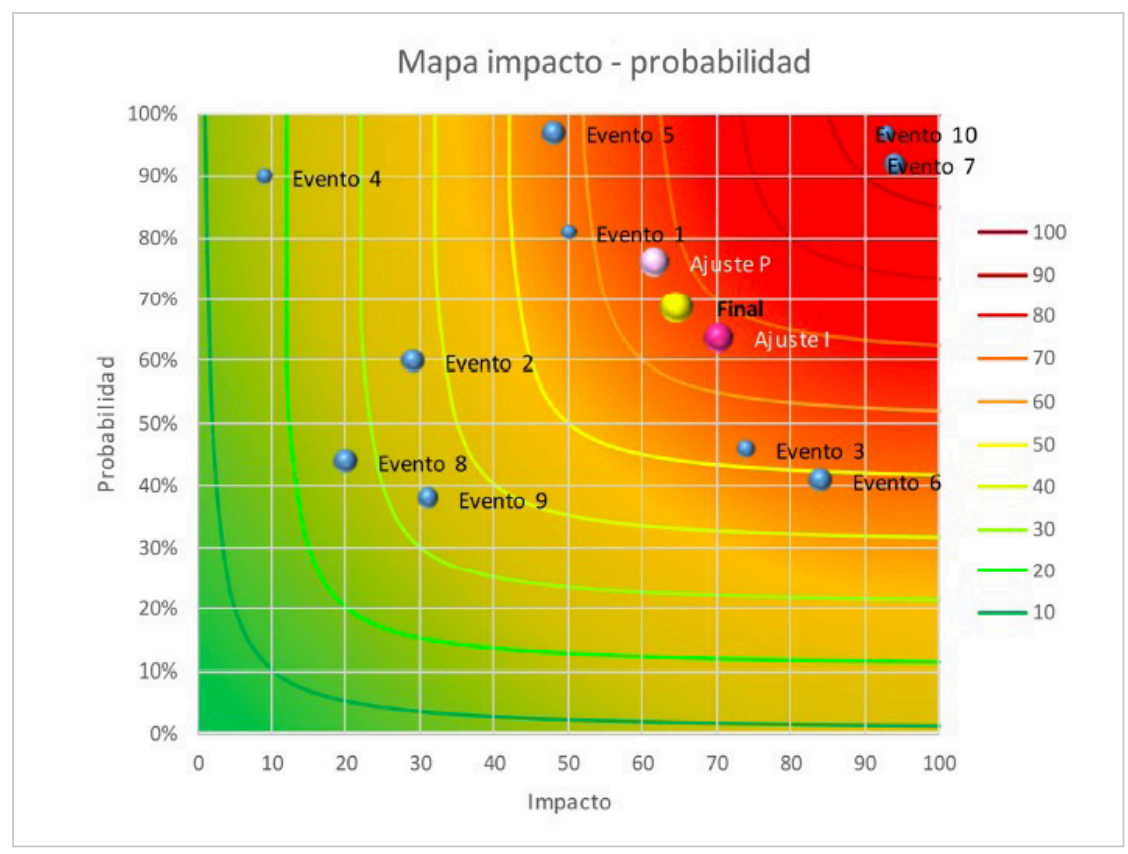

Figura 4. Puntos de ajuste y combinación de riesgos 


$$
\delta=\frac{F v_{m}}{F v_{a}}
$$

y, por tanto:

$$
I_{f}=\delta \times I_{m a}, P_{f}=\delta \times P_{m a} . F v_{f}=F v\left(I_{f}, P_{F}\right)=F v_{m}
$$

La figura 4 muestra gráficamente el proceso descrito. El tamaño de los puntos es proporcional a su peso o ponderación individual. Puede comprobarse que los puntos de ajuste están próximos y en la misma curva de nivel Fv. El punto final, de color amarillo, es el promedio entre ambos y es el resultado buscado.

\section{El Factor de visibilidad Fv es el resultado de la investigación realizada para obte- ner un indicador lineal, intuitivo y fácil de comprender}

Estos cuestionarios son aplicables en proyectos de construcción; para otros sectores habría que adaptarlos.

Cada pregunta, cada sección y cada cuestionario tiene un peso que se utiliza al combinarlos. Estos pesos se definen como variables en función de parámetros del proyecto: relevancia, país, presupuesto, tipo de cliente, tipo de contrato y emplazamiento.

La información comunicada debe ser clara, objetiva y fácilmente comprensible

No es fácil que sucedan simultáneamente todos los riesgos posibles, pero puede haber varios cientos y una descripción pormenorizada de todos ellos para comprender la situación de conjunto no sería satisfactoria. Con la formulación presentada anteriormente se puede obtener el resumen que se muestra en la figura 5 .

\subsection{Definición de cuestionarios}

El uso de una lista de comprobación con preguntas y respuestas predeterminadas asegura que no se habrá dejado de considerar por olvido ninguno de esos riesgos, pero es excluyente. Sin embargo el método utilizado para su definición garantiza que posteriormente no surgirán riesgos relevantes no disponibles en el cuestionario.

Hay muchas publicaciones acerca de posibles clasificaciones de los riesgos (Mishra; Mishra, 2016) y su enfoque es identificar los orígenes y elaborar una estructura en árbol. La investigación realizada mostró que en la práctica es preciso considerar la estructura organizativa de la empresa cuyos proyectos se examinan, para organizar los riesgos y distribuir los cuestionarios. Además, cada departamento (legal, técnico, logístico, etc.) conoce las materias que les atañen y qué riesgos relativos a ellas se presentan más frecuentemente en los proyectos.

Se definieron 12 cuestionarios organizados en secciones, subsecciones, preguntas y respuestas (ver tabla 2).

La tabla 2 indica el número de posibles respuestas de riesgo. Los dos últimos cuestionarios no llegaron a ponerse en producción.

El total de preguntas es de 528 y el de respuestas 1.726 . Las preguntas definen genéricamente el riesgo a valorar y las respuestas su detalle, no requiriéndose narración.
Tabla 2. Estructura de los cuestionarios

\begin{tabular}{|l|c|c|c|c|}
\hline Cuestionarios & Secciones & Subsecciones & Preguntas & Respuestas \\
\hline País & 11 & - & 73 & 73 \\
\hline Jurídico & 18 & - & 32 & 55 \\
\hline Contrato & 11 & - & 26 & 107 \\
\hline Comercial & 6 & 3 & 63 & 66 \\
\hline Financiero & 6 & 2 & 29 & 119 \\
\hline Técnico & 7 & 61 & 128 & 589 \\
\hline Recursos humanos & 4 & - & 9 & 37 \\
\hline Logístico & 3 & 15 & 42 & 160 \\
\hline Medioambiental & 3 & - & 15 & 52 \\
\hline PRL (prevención de riesgos laborales) & 1 & - & 4 & 24 \\
\hline Deber de protección & 3 & - & 11 & 60 \\
\hline Tecnologías de la información & 7 & 16 & 96 & 384 \\
\hline
\end{tabular}

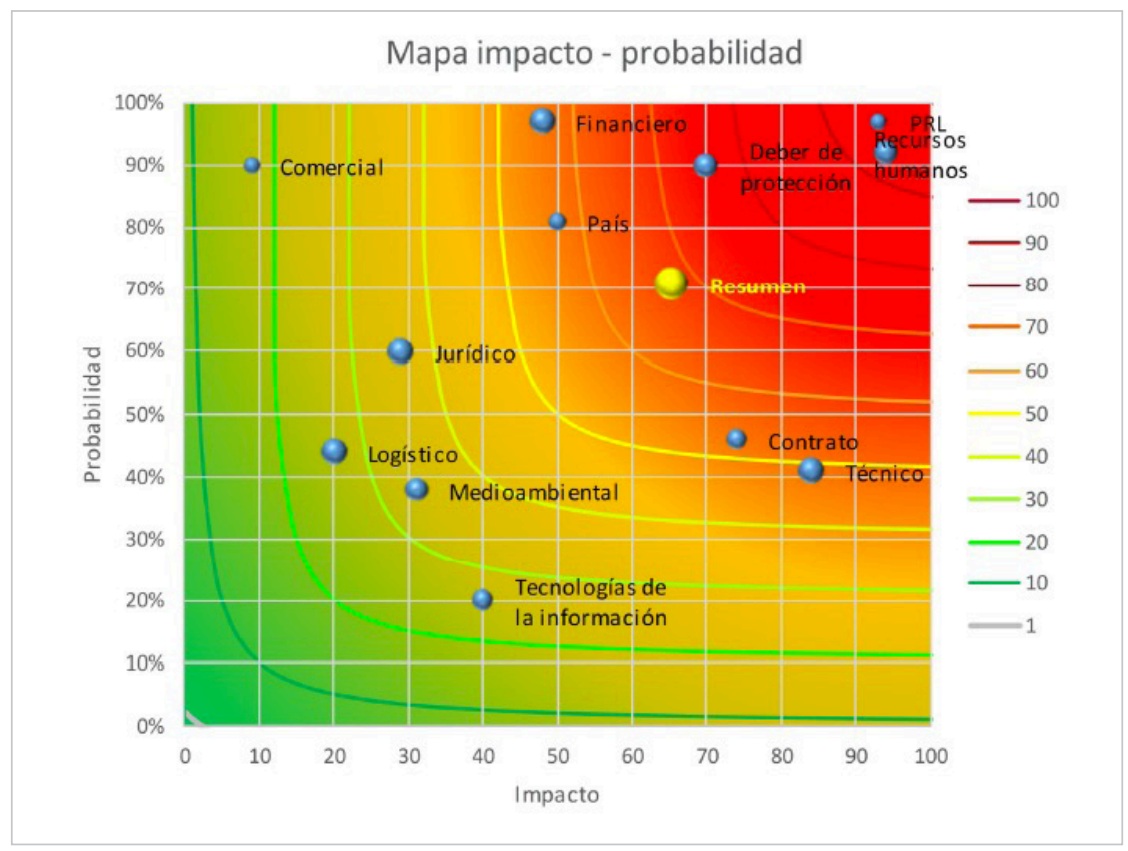

Figura 5. Resumen de riesgos de un proyecto por cuestionarios y total 
Tabla 3. Campos para la identificación y valoración de un riesgo y condición de visibilidad

\begin{tabular}{|l|l|l|l|}
\hline \multicolumn{1}{|c|}{ Campo } & \multicolumn{1}{c|}{ Tondición } & \multicolumn{1}{c|}{ Explicación } \\
\hline Impacto & Riesgo identificado & Desplegable & Valoración cualitativa impacto \\
\hline Probabilidad & Riesgo identificado & Desplegable & Valoración cualitativa probabilidad \\
\hline Tipo de impacto & Riesgo identificado & Desplegable & Coste, plazo, reputación, etc. \\
\hline Coste base de cálculo & Riesgo identificado & Numérico & Estimación coste daño producido \\
\hline Respuesta & Riesgo identificado & Desplegable & Aceptar, mitigar, evitar, transferir \\
\hline Medida mitigadora & Mitigar & Texto & Descripción \\
\hline Tipo medida mitigación & Mitigar & Desplegable & Negociación, técnica, organizativa, etc. \\
\hline Coste medida mitigación & Mitigar & Numérico & Estimación coste medida mitigadora \\
\hline Momento mitigación & Mitigar & Desplegable & Antes firma, inicio proyecto, etc. \\
\hline$\%$ impacto remanente & Mitigar & Desplegable & \% impacto remanente sobre original \\
\hline$\%$ probabilidad remanente & Mitigar & Desplegable & \% probabilidad remanente sobre original \\
\hline Comentarios & - & Texto & Comentarios adicionales \\
\hline
\end{tabular}

\subsection{Definición de campos y lógica de introducción de datos}

El sistema propuesto elimina casi completamente la redacción de texto, y facilita al evaluador interactuar inteligentemente con el cuestionario -según se adelantó en el apartado 3.1.1. Diseño- para que su atención se dedique exclusivamente a la valoración de los riesgos (Card; Mackinlay; Shneiderman, 1999; Olmeda-Gómez, 2014). Los campos se muestran sólo cuando es preciso en función de los datos que va introduciendo el evaluador (tabla 3).

El evaluador actúa con el ratón y no escribiendo, por lo que no se guarda texto sino datos

los campos de cada riesgo (tabla 4).

Los valores correspondientes a la situación mitigada se muestran numérica y gráficamente. Los puntos correspondientes a los resúmenes mitigados de cuestionarios no se dibujan por razón de claridad visual (figura 6).

\subsection{Definición del sistema de va- loración del coste de los riesgos}

La determinación del coste individual de cada riesgo y la estimación del coste total probable para el proyecto tienen una complejidad que, aunque ha debido ser estudiada para la definición del sistema de riesgos propuesto, queda fuera del alcance de este artículo.

Sin entrar en los detalles del cálculo numérico, el planteamiento es visual (Card; Mackinlay; Shneiderman, 1999; Olmeda-Gómez, 2014). La no-
Tabla 4. Campos calculados para cada riesgo

\begin{tabular}{|l|l|}
\hline \multicolumn{1}{|c|}{ Campo } & \multicolumn{1}{c|}{ Explicación } \\
\hline FV & Valor del Factor de visibilidad \\
\hline Coste probable actual & Coste base x probabilidad \\
\hline Coste probable mitigado & Coste riesgo mitigado + coste medida \\
\hline
\end{tabular}

vedad es huir del enfoque determinista habitual y presentar el coste del riesgo como una franja de posibles importes a considerar que oriente al estimador. Esta franja se calcula automáticamente considerando los siguientes valores:

- Coste base de cálculo

- Coste medida mitigación

- \%impacto remanente

- \%probabilidad remanente

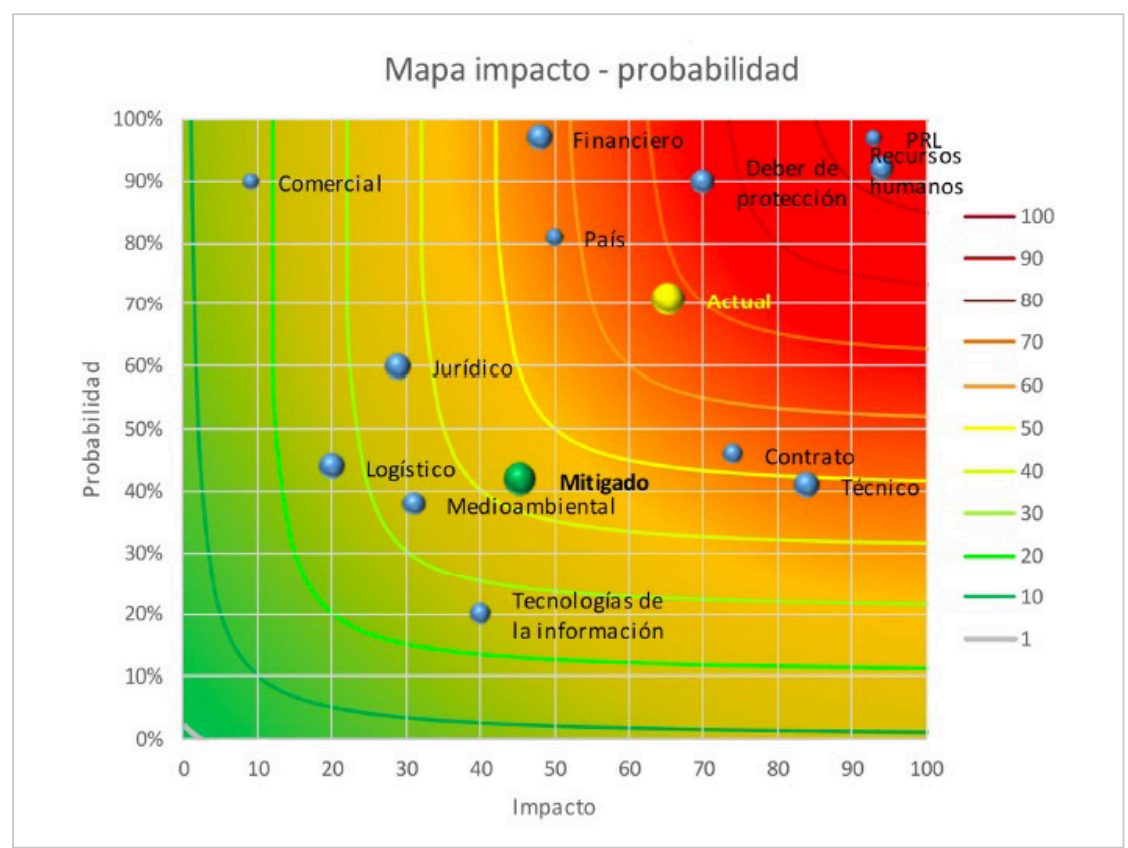

Figura 6. Efecto de la mitigación de riesgos de un proyecto 
Con estos datos se calculan automáticamente los límites inferior y superior de coste del riesgo. El coste real será mayor por cuestiones probabilísticas y porque no es posible estimar el coste de muchos riesgos. Al estimador se le presentan estos valores y se introduce el concepto de mejora, que es la diferencia entre la situación inicial y la situación mitigada. La mejora define el beneficio conseguible mediante una correcta mitigación del riesgo, según muestra la figura 7.

\section{Discusión y conclusiones}

Se indicaba al inicio de este artículo que los informes de riesgo de un proyecto contienen decenas de páginas, al igual que los informes ejecutivos de situación global, y que era un reto resumir esa información con pocas palabras.

Con el sistema propuesto se resume la información esencial de riesgos de un proyecto en una sola hoja, gracias al uso del Factor de visibilidad (figura 8).

La hoja resumen muestra el mapa de calor con el resumen de cada cuestionario y las situaciones globales actual y mitigada. Además, detalla en una tabla los valores de impacto, probabilidad, Factor de visibilidad y coste para cada cuestionario antes y después de mitigar, utilizando un código de colores. Se muestran los 10 riesgos destacados, según su Factor de visibilidad y su coste, las posibilidades de mejora correspondientes a cada cuestionario y el desglose de posibles ahorros clasificados por tipo de mitigación.

El sistema propuesto proporciona visibilidad de los países con mayor riesgo en función de sus proyectos (figura 9).

El analista o responsable puede visualizar interactivamente la información de riesgo total, por zona geográfica, país o pro-

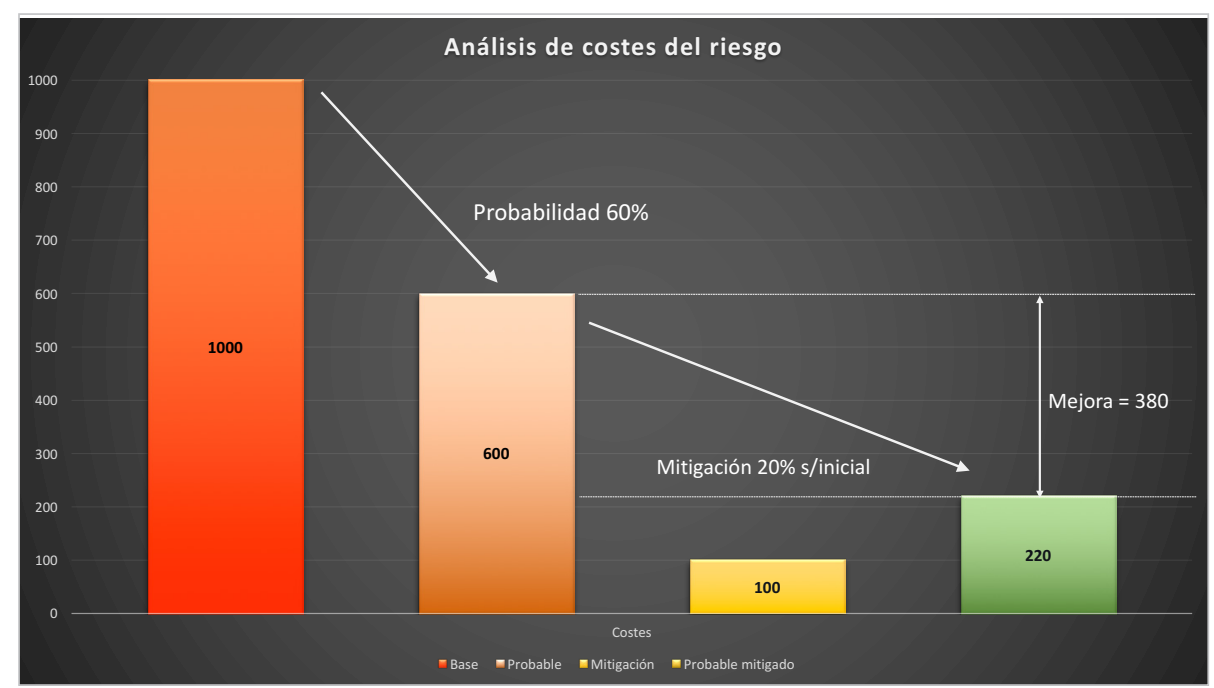

Figura 7. Ejemplo visual de esquema de costes de riesgo de un proyecto
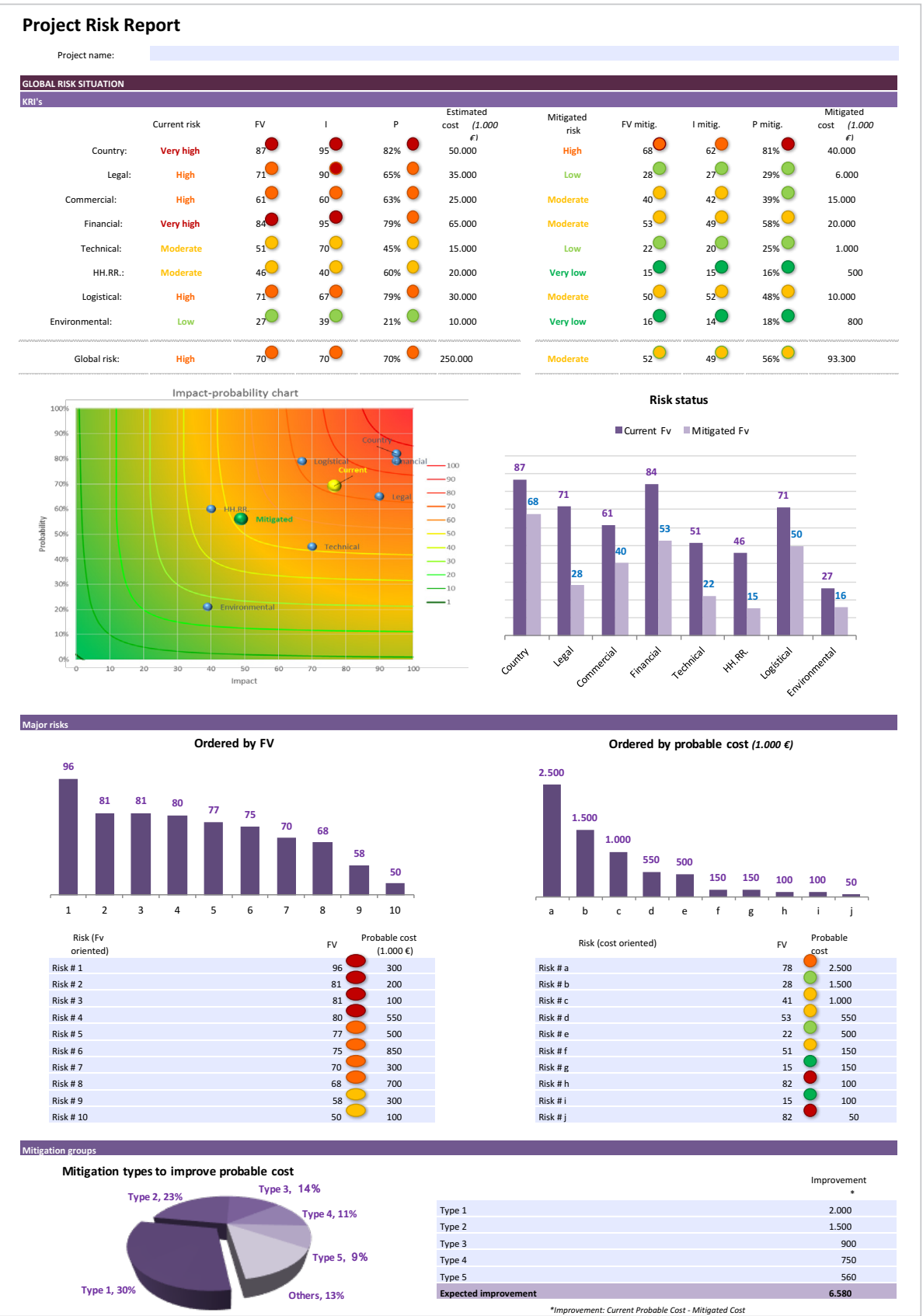

Figura 8. Ejemplo de hoja resumen de riesgos de un proyecto 


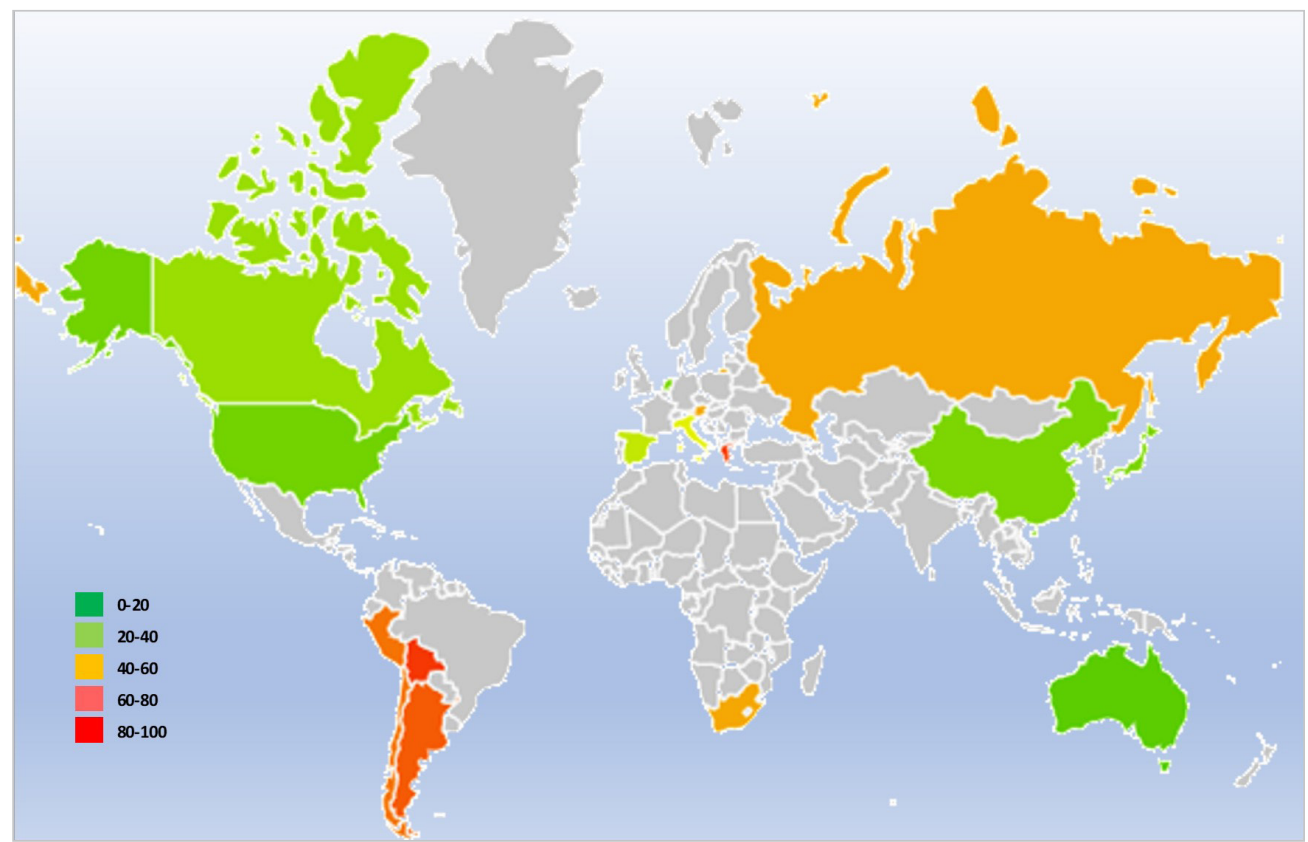

Figura 9. Visión general de los riesgos de proyecto por países (datos ficticios)

yecto, seleccionando el elemento para el que quiere ver los detalles (figuras 10 y 11).

\section{Con el sistema propuesto se resume la información esencial de riesgos de un proyecto en una sola hoja, gracias al uso del Factor de visibilidad}

El sistema presentado significa un gran avance en el tratamiento de la información de riesgos. Añade objetividad, aportando un indicador cuantitativo automático que es genérico, lineal e intuitivo, que posibilita el resumen y la comparación, y que sintetiza la información para facilitar su análisis, destacando la más relevante. Crea un estándar aplicable a la mayor parte de las empresas, tanto para los riesgos de sus proyectos como para su riesgo global.

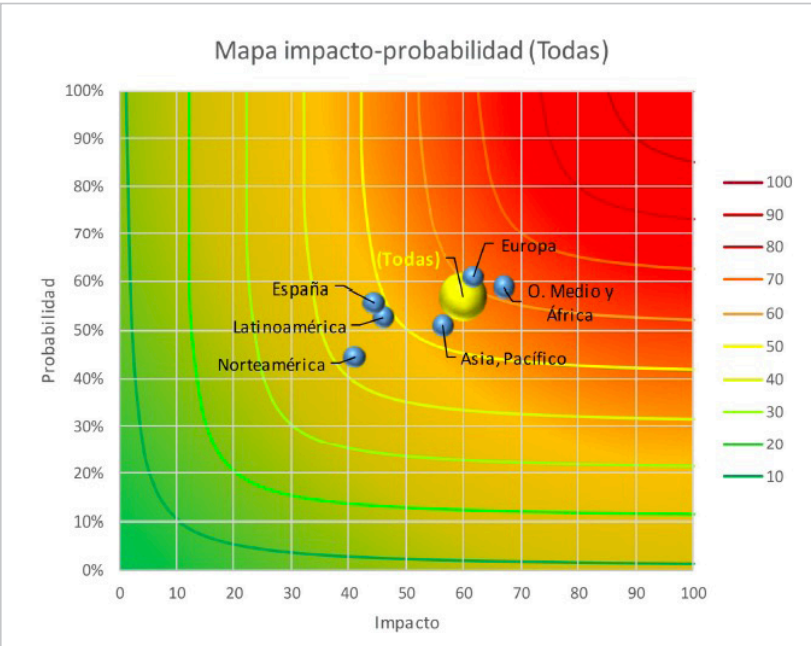

Figura 10. Visión general de los riesgos de proyecto por zonas geográficas (datos ficticios)
El indicador de Factor de visibilidad, y su método de combinación aportan una claridad y flexibilidad alejadas de las limitaciones de los sistemas tradicionales. Su orientación visual facilita la rápida identificación de los riesgos principales y la comprensión del riesgo global del escenario considerado.

\section{Agradecimientos}

El autor agradece a la Escuela Técnica Superior de Ingenieros de Caminos, Canales y Puertos de Madrid (Universidad Politécnica de Madrid) y a su director de tesis Fernando Rodríguez López, el contexto investigador necesario para la realización de este artículo, y a la directora de la biblioteca Concepción García-Viñuela su apoyo y ayuda para obtener algunos de los artículos estudiados. Adicionalmente el autor agradece a Katherine Reddington su inestimable revisión del abstract.

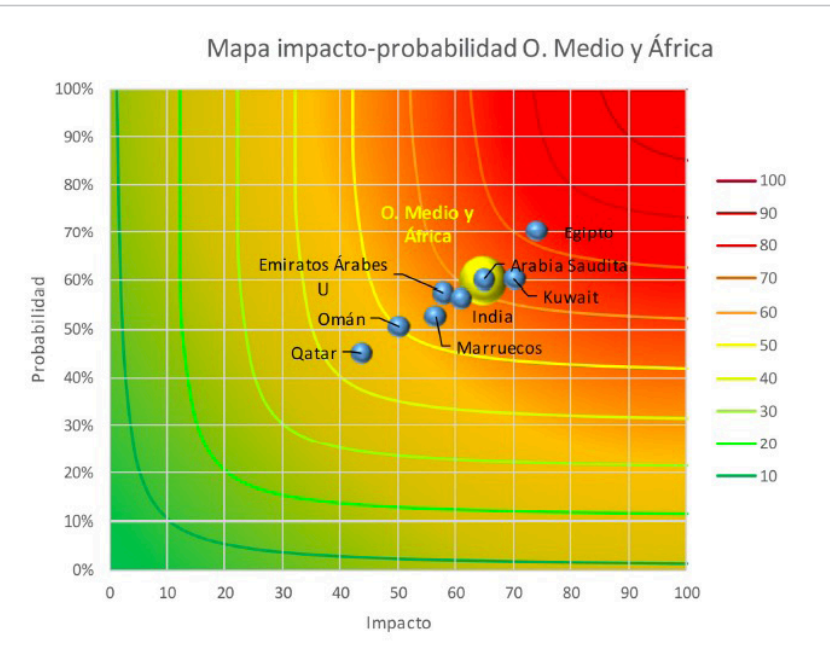

Figura 11. Visión de los riesgos de proyecto por países de una zona geográfica (datos ficticios) 


\section{Referencias}

Aenor (2010). Gestión del riesgo. Principios y directrices. Términos y definiciones. UNE-ISO 31000:2010. Aenor, 28 julio. http://www.aenor.es/aenor/normas/normas/fichanorma. asp ?tipo $=N \&$ codigo $=N 0045825$

Aenor (2011). Gestión del riesgo. Técnicas de apreciación del riesgo. UNE-EN 31010:2011. Aenor, 5 abril.

http://www.aenor.es/aenor/normas/normas/fichanorma. asp?tipo $=N \&$ codigo $=N 0047287$

Airmic/Alarm/IRM (2010). A structured approach to enterprise risk management (ERM) and the requirements of ISO 31000.

http://www.ferma.eu/app/uploads/2011/10/a-structuredapproach-to-erm.pdf

Al-Bahar, Jamal F.; Crandall, Keith C. (1990). "Systematic risk management approach for construction projects". Journal of construction engineering and management, v. 116, n. 3, pp. 533-546.

https://goo.gl/mxbxJn

https://doi.org/10.1061/(ASCE)0733-9364(1990)116:3(533)

Arjona, Álvaro (2015). La función de auditoría interna, la gestión de riesgos y el control interno: motores del cambio en las empresas del siglo XXI. Madrid: CEU Universidad San Pablo.

Babatola, Mayowa (2004). Developing a KRI program: Guidance for the operational risk manager. BITS. Financial Services Roundtable.

http://fsroundtable.org/wp-content/uploads/2015/07/ KRIprogram0904.pdf

Beasley, Mark S.; Branson, Bruce C.; Hancock, Bonnie V. (2010). Developing key risk indicators to strengthen enterprise risk management. How key risk indicators can sharpen focus on emerging risks. Durham, USA: COSO.

https://www.coso.org/Documents/COSO-KRI-Paper-FullFINAL-for-Web-Posting-Dec110-000.pdf

Card, Stuard K.; Mackinlay, Jock D.; Shneiderman, Ben (1999). Readings in information visualization: using vision to think. San Francisco, CA, USA: Morgan Kaufmann. ISBN: 15 58605339

COSO (2004). Gestión de riesgos corporativos. Marco integrado. Técnicas de aplicación. USA: Committee of Sponsoring Organizations of the Treadway Commission, COSO.

https://goo.gl/fxjvte

Cretu, Ovidiu; Stewart, Robert; Berends, Terry (2011). Risk management for design and construction. Hoboken, New Jersey, USA: Wiley. ISBN: 9780470635384 https://doi.org/10.1002/9781118984017

Curtis, Patchin; Carey, Mark (2012). Risk assessment in practice. USA: Committee of Sponsoring Organizations of the Treadway Commission, COSO.

https://goo.gl/2Vpk4W

De-Frutos-Torres, Belinda; Marcos-Santos, Mercedes (2017). "Disociación entre las experiencias negativas y la percepción de riesgo de las redes sociales en adolescentes". El profesional de la información, v. 26, n. 1, pp. 88-96. https://doi.org/10.3145/epi.2017.ene.09

Di-Marco, Melissa; Taylor, John; Alin, Pauli (2010). “Emergence and role of cultural boundary spanners in global engineering project networks". Journal of management in engineering, v. 26, n. 3, pp. 123-132.

https://goo.gl/WyzJFh

https://doi.org/10.1061/(asce)me.1943-5479.0000019

Ferma; Eciia (2014). Audit and risk committees - News from EU legislation and best practices. Ferma; Eciia.

http://www.ferma.eu/app/uploads/2014/10/ECIIA_ FERMA_Brochure_v8.pdf

Leung, Mei-Yung; Liang, Qi; Chan, Isabelle Y. S. (2017). “Development of a stressors-stress-performance-outcome model for expatriate construction professionals". Journal of construction engineering and management, v. 143, n. 5. https://doi.org/10.1061/(asce)co.1943-7862.0001266

Likert, Rensis (1932). "A technique for the measurement of attitudes". Archives of psychology, v. 22, n. 140, pp. 5-55. https://legacy.voteview.com/pdf/Likert_1932.pdf

Lundy, Duane E. (2012). "Degrees of quality: A method for quantifying aesthetic impact". Psychology research, v. 2, n. 4, pp. 205-221.

https://dx.doi.org/10.17265/2159-5542/2012.04.001

Ministerio de Economía y Competitividad (2016). Informe mensual de comercio exterior, diciembre de 2015. Ministerio de Economía y Competitividad - Gobierno de España. http://www.mineco.gob.es/stfls/mineco/prensa/ficheros/ noticias/2016/160218_Informe_COMEX_Diciembre_15.pdf

Mishra, Surabhi; Mishra, Brajesh (2016). "A study on risk factors involved in the construction projects". International journal of innovative research in science, v. 5, n. 2, pp. 11901196.

https://dx.doi.org/10.15680/IJIRSET.2016.0502005

https://www.ijirset.com/upload/2016/february/5_A\%20 Study.pdf

Olmeda-Gómez, Carlos (2014). "Visualización de información". El profesional de la información, v. 23, n. 3, pp. 213220.

https://doi.org/10.3145/epi.2014.may.01

Pont-Sorribes, Carles; Codina, Lluís; Pedraza-Jiménez, Rafael (2009). "Comunicación de riesgo y sistemas de información en la Web: cinco modelos". El profesional de la información, v. 18, n. 4, pp. 389-397.

https://doi.org/10.3145/epi.2009.jul.05

Pratt, John W. (1964). "Risk aversion in the small and in the large". Econometrica, v. 32, n. 1, pp. 122-136.

http://www2.econ.iastate.edu/classes/econ642/Babcock/ pratt.pdf

https://doi.org/0012-9682(196401/04)32:1/2<122:RAITSA>2. $0 . C O ; 2-W$

Project Management Institute (2013). Guía de los fundamentos para la dirección de proyectos (Guía del PMBOK-Quinta edición). NewTown Square, Pensilvania, USA: Project Management Institute, Inc. ISBN: 9781628250091 https://www.gob.mx/cms/uploads/attachment/file/79535/ PMBOK_5ta_Edicion_Espanol_1_.pdf 
Schuyler, John (2001). Risk and decision analysis in projects. Princeton, New Jersey, USA: Project Management Institute, Inc. ISBN: 1880410281

Seopan (2015). "Informe económico 2014". Seopan. https://goo.gl/4jSdZA

Steinberg, Richard M.; Everson, Miles E. A.; Martens, Frank J.; Nottingham, Lucy E. (2004). "Enterprise risk management - integrated framework. Executive summary". USA: coso.

https://www.coso.org/Documents/COSO-ERM-ExecutiveSummary.pdf

The Institute of Risk Management (2011). Risk appetite and tolerance - Executive summary. The Institute of Risk Management.

https://goo.gl/mq1rpP

The Institute of Risk Management (2012). Risk culture under the microscope. Guidance for boards. The Institute of Risk Management.

https://www.theirm.org/media/885907/Risk_Culture_A5_ WEB15_Oct_2012.pdf

The Joint Forum (2003). Trends in risk integration and aggregation. Basel Committee on Banking Supervision; International Organization of Securities Commissions; International Association of Insurance Supervisors. http://www.bis.org/publ/joint07.pdf
Vilajoana-Alejandre, Sandra; Rom-Rodríguez, Josep (2017). "Sistema de autorregulación publicitaria: del compromiso ético al control efectivo de la publicidad en España". El profesional de la información, v. 26, n. 2, pp. 192-200. https://doi.org/10.3145/epi.2017.mar.05

Whittaker, Margaret H. (2015). "Risk assessment and alternatives assessment: Comparing two methodologies". Risk analysis, v. 35, n. 12, pp. 2129-2136.

https://doi.org/10.1111/risa.12549

World Economic Forum (2015). Global risks 2015. $10^{\text {th }}$ edition. Geneva, Switzerland: World Economic Forum.

http://reports.weforum.org/global-risks-2015

World Economic Forum (2016). The global risks report 2016. $11^{\text {th }}$ edition. Geneva, Switzerland: World Economic Forum. http://reports.weforum.org/global-risks-2016

World Economic Forum (2017). The global risks report 2017. $12^{\text {th }}$ edition. Geneva, Switzerland: World Economic Forum. ISBN: 9781944835071

http://reports.weforum.org/global-risks-2017

Zou, Patrick X. W.; Chen, Ying; Chan, Tsz-Ying (2010). “Understanding and improving your risk management capability: Assessment model for construction organizations". Journal of construction engineering and management, v. 136, $n$. 8, pp. 854-863.

https://doi.org/10.1061/(ASCE)CO.1943-7862.0000175

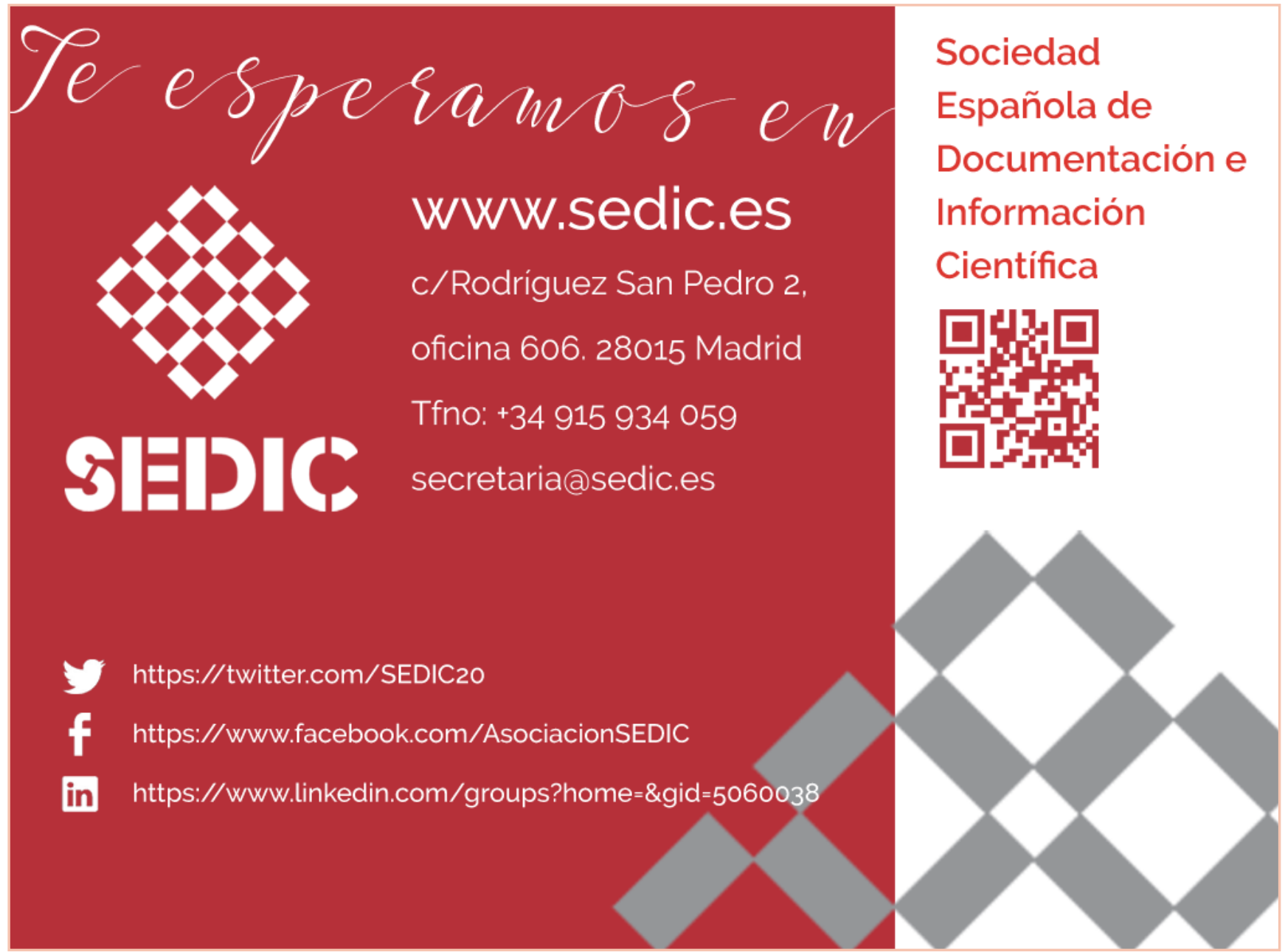

